\title{
Antiphase domain growth: Correlation functions and structure factors in the scaling regime
}

\author{
Ulrike Zweck $\odot^{*}$ and Michael Leitner $\oplus^{\dagger}$ \\ Technische Universität München, Physik-Department and Forschungs-Neutronenquelle Heinz Maier-Leibnitz, (FRM II), \\ 85748 Garching, Germany
}

(Received 11 June 2021; accepted 2 August 2021; published 2 September 2021)

\begin{abstract}
We study the coarsening of three-dimensional antiphase domain structures via Monte Carlo simulations. Linear lattice dimensions of $N=1024$ enable us to reach a scaling regime covering about 2.5 orders of magnitude of linear scale. With short-range interactions on cubic lattices at temperatures of $0.75 T_{\mathrm{c}}$, the resulting antiphase domain structures are isotropic, which allows us to describe the real-space correlation functions by a common function scaled by a time-dependent parameter. We compare abstract Potts models and realistic models of atomic order in compounds and show that those with same ground-state degeneracy $q$ lead to equivalent antiphase domain structures, while the scaling functions for different $q$ show slight but significant deviations. Finally, we quantitatively discuss notions of real-space scale (specific interface area) and reciprocal-space scale (superstructure peak width) and thus give numerically exact values for the corresponding parameter $K$ of the Scherrer equation.
\end{abstract}

DOI: 10.1103/PhysRevB.104.094101

\section{INTRODUCTION}

Ordering transitions in compounds, where the lattice of $a$ priori equivalent sites decays into sublattices with preferred occupations by different elements, can have profound effects on structural as well as functional materials properties [1-3]. For instance, the increased size of the unit cell implies larger minimal Burgers vectors of dislocations and thus increases the yield strength of ordered compounds considerably over their disordered states [4]. Superalloys, such as $\mathrm{Ni}_{3} \mathrm{Al}$ and $\mathrm{Fe}_{3} \mathrm{Al}$ or the lightest intermetallic ordered compound $\mathrm{Al}_{3} \mathrm{Li}$ with a $L 1_{2}$ superstructure on the fcc lattice, display remarkable mechanical strength at a low density $[1,2,5]$.

Due to the spontaneous breaking of symmetry, the superstructures nucleated at independent regions in the crystal do not necessarily fit together. After the ordered phase has grown to cover the whole crystal, the resulting interfaces divide the crystal into so-called antiphase domains (APDs) and are correspondingly called antiphase domain boundaries. With increasing annealing time the domains start to coarsen, reducing the excess free energy of the domain walls and increasing the material's hardness [6].

Experimentally the size of domains is accessible either by real-space methods, e.g., electron microscopy, or by diffraction methods in reciprocal space, perfectly analogous to the more prominent case of crystallite sizes in polycrystalline

\footnotetext{
*ulrike.zweck@frm2.tum.de

${ }^{\dagger}$ michael.leitner@frm2.tum.de
}

Published by the American Physical Society under the terms of the Creative Commons Attribution 4.0 International license. Further distribution of this work must maintain attribution to the author(s) and the published article's title, journal citation, and DOI. media. In microscopy the APD scale is most commonly [7-9] evaluated using the linear intercept method [10], where the characteristic size is determined as the mean distance between intersections with APD boundaries along a curve of random direction [10].

In diffraction, the finite correlation length of the superstructure due to the APD boundaries gives rise to a broadening of the superstructure peaks. Here the easiest approach is to consider descriptive parameters such as the full width at half maximum (FWHM) or the integral breadth (the quotient of peak area and peak height) that is according to the Scherrer equation [11] inversely proportional to the domain size $\bar{D}=$ $2 \pi K / \Delta k$. In more involved evaluations, a heuristic shape and size distribution function is Fourier transformed and modeled to the powder pattern [12]. In any case, the shape of the domain configuration has to be taken into account, either as a shape constant parameter $K$ as in case of the Scherrer equation or a as model for the peak shape. Since the statistical properties of APD structures have no closed form, the determined scale parameters are specific to the analysis method and the used assumptions. In particular, in most cases domain sizes determined by diffraction cannot be related to specific real-space features of the APD configuration (but see, e.g., Refs. [13-15] for past approaches to this problem).

The starting point of typical theoretical treatments of domain coarsening is to average over the atomic scale with its discreteness and disorder, so that the resulting domain pattern completely specifies the system's configuration. In the absence of microscopic randomness, its evolution is deterministic and dictated by interface curvature according to the classical Allen-Cahn treatment [16]. At late times, the evolution equations can be linearized, so that, if we have two configurations $\sigma_{1}(\vec{r})$ and $\sigma_{2}(\vec{r})$ that are identical after rescaling the spatial domain $\sigma_{2}(\vec{r})=\sigma_{1}(\lambda \vec{r})$, then, if $\sigma_{1}(\vec{r})$ evolves into $\sigma_{1}^{\prime}(\vec{r})$, also $\sigma_{2}(\vec{r})$ will evolve into $\sigma_{2}^{\prime}(\vec{r})=\sigma_{1}^{\prime}(\lambda \vec{r})$, albeit after 
a correspondingly rescaled time interval. The scaling hypothesis in the strict sense of the word [17] now corresponds to the assumption that there is an attractive fixed point in the space of spatially rescaled statistical properties of domain configurations to which a typical configuration will converge. As a consequence, the single characteristic length scale will show a power-law dependence on time-for the specific case of curvature-driven coarsening of nonconserved fields with it should possess an exponent of $v=1 / 2$.

If only the growth kinetics are the focus of the study, the scaling behavior of the structure factor $S(k, t)$ can be exploited [18], casting the structure factor in the form of $S(k, t)=L(t)^{3} S(k L(t))$, with the time dependent scaling factor $L(t)[17,19]$. The Fourier transform of the structure factor is the pair-correlation function, which consequently obeys the corresponding scaling behavior $g(r, t)=g(r / L(t))$.

The assumption of infinitely sharp domain interfaces leads to the correlation function behaving like $g(r) \approx 1-\alpha r$ at small distances, with $\alpha$ being proportional to the specific interface area $\sigma$ [20]. This small-scale linear decay with a discontinuous derivative at the origin when seen as a function of three-dimensional real space corresponds to the so-called Porod's law $S(k) \propto k^{-4}$ for the scaled structure factor at large $k$ [21], which can conveniently be probed via the small-angle scattering in phase-separation processes [22]. In contrast, this large- $k$ regime with its simple behavior is typically not accessible for broadened superstructure peaks in ordering processes, necessitating a detailed treatment of the full peak shape.

There have been different approaches to theoretically derive the scaled correlation function, or equivalently the reciprocal-space structure factor [19,23-26] (for a comparison of the fundamental assumptions and approximations see Ref. [17]). The predictions do not differ much and are in general agreement with experiment. On the other hand, numerical simulations of coarsening processes with nonconserved order parameters are plentiful, but typically concentrate on cases with unusual behavior such as the two-dimensional $O(2)$ model (e.g., Ref. [27]) with its Berezinskii-KosterlitzThouless transition [28,29]. We are aware of only two simulations of discrete symmetry breaking with small groundstate degeneracy in three dimensions, the case relevant for APD coarsening, but these treated only small systems with correspondingly short scaling regimes, and only the twodomain case [30,31].

Our aim in this article is twofold: First, we will present large-scale simulations of antiphase domain coarsening with up to four inequivalent domains in three dimensions, treating both microscopically realistic as well as abstract Potts models. Second, we will propose a general model for the real-space correlation function and, as its Fourier transform, the reciprocal-space structure factor, and determine the set of parameters that give a very satisfactory fit to our Monte Carlo simulations. In addition we will show that, as long as interactions are short range and give rise to approximately isotropic APD interface energies, the obtained scaling functions do not depend on the specific microscopic model but are universal with a universality class determined by the ground-state degeneracy of the ordered state, which opens the possibility to quantitatively relate the length scales determined by real- space microscopy to those determined by the broadening of diffraction peaks.

\section{SYSTEMS}

In order to have approximately isotropic APD interface energies, the cubic symmetry of the lattice is of course a precondition. We consider here cubic systems with twofold, threefold, and fourfold degenerate ground states, each in two levels of detail, which in the following we will term the microscopic and the abstract models, respectively:

The microscopic models correspond to Ising models at given stoichiometry, where ordering and APD coarsening proceed via the movement of a vacancy and the corresponding local shuffling of atoms. Thus, the number of atoms of a given kind is conserved, while the ordering parameter, corresponding to the assignment of specific kinds of atoms to sublattices, is not conserved.

The abstract models with $q$-fold ground-state degeneracy correspond just to the standard $q$-state Potts models [32]. In this case, the values of the spins, which are the fundamental degrees of freedom in the simulation, correspond directly to the domain a given site belongs to. Thus, the number of spins of a given kind is not conserved.

Note that for $q=2$, the microscopic (Ising) and abstract (Potts) models are equivalent in terms of thermodynamics, but not in terms of dynamics. Thus, we simulated the two cases independently to serve as a check for our implementations. On the other hand, all microscopic systems we are aware of with $q>4$ host qualitatively different kinds of APD boundaries and are thus beyond our scope, while the corresponding abstract models would of course still be amenable to our approach.

\section{A. Microscopic models}

In general we consider a Hamiltonian of

$$
H=-J_{\mathrm{nn}} \sum_{\langle i, j\rangle} \sigma_{i} \sigma_{j}-J_{\mathrm{nnn}} \sum_{\langle i, j\rangle^{\prime}} \sigma_{i} \sigma_{j} .
$$

Here $\langle i, j\rangle$ and $\langle i, j\rangle^{\prime}$ denote the summation over all nearestneighbor and next-nearest-neighbor pairs of sites, respectively, with each pair counted once. The spin variables $\sigma_{i}$ are either +1 or -1 . In the following we always choose the scale of energy so that $J_{\mathrm{nn}}=-1$, leading to a preference for unlike nearest-neighbor pairs.

For twofold ground-state degeneracy, we use Eq. (1) on a simple-cubic lattice with equal concentration of the two kinds of atoms. We choose $J_{\mathrm{nnn}}=0$, giving the standard threedimensional nearest-neighbor antiferromagnetic Ising model (which of course is thermodynamically equivalent to the ferromagnetic Ising model). Its ground state corresponds to the B1 superstructure (also known as the $\mathrm{NaCl}$ structure), obtained by assuming the space group $225(F m \overline{3} m)$ and assigning $\mathrm{A}$ and $\mathrm{B}$ atoms to the Wyckoff positions $4 \mathrm{a}$ and $4 \mathrm{~b}$, respectively. With two kinds of domains, there is only one APD boundary, the nearest-neighbor bonds between the lattice sites give rise to a bipartite graph, and due to the symmetry of the Hamiltonian, there will be no segregation to the boundaries. In this sense, this system is the simplest and thus arguably the most popular 

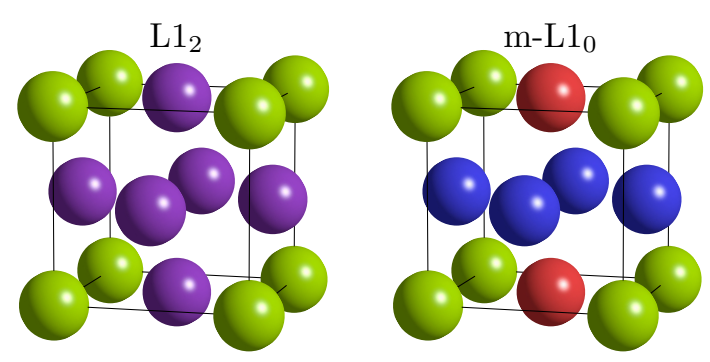

FIG. 1. The two microscopic models as superstructures on the fcc lattice: $L 1_{2}$ (left) and $\mathrm{m}-L 1_{0}$ (right). The freedom in choosing on of the four simple cubic sublattices as the green minority sublattice in $L 1_{2}$ results in $q=4$ degeneracy, while an ordering of the red and blue atoms to $\mathrm{m}-L 1_{0}$ results in additional $q=3$ degeneracy.

to study APD structures theoretically [30,33]. Note that the corresponding case on the body-centered cubic lattice with the B2 ground state is qualitatively equivalent and the subject of numerous experimental studies [34-36].

The prototypical case for fourfold ground-state degeneracy is given by the $L 1_{2}$ structure $\left(\mathrm{Cu}_{3} \mathrm{Au}\right)$ with space group 221 $(P m \overline{3} m)$ and the two kinds of atoms occupying the (3c) and (1a) sites, respectively (Fig. 1). Again we assume perfect $3: 1$ stoichiometry. In the idealized case, it is well known that for any two given APDs, the boundary energy is strongly anisotropic, as there is one among the three $\{100\}$ plane families that allows a boundary while keeping all the nearestneighbor relations unchanged [13]. As a result, particularly at low temperatures, the simulated APDs are faceted strongly in these directions [37]. Since the coarsening of domains is a curvature-driven process, the domain growth is slowed down, so that instead of the predicted [16] and experimentally obtained [38] growth exponent of $v=\frac{1}{2}$, an effective exponent of $v \approx \frac{1}{4}$ is observed in simulations [37,39]. By including a positive next-nearest-neighbor interaction $J_{\mathrm{nnn}}$, this behavior can be suppressed [40]. Experimentally, the full range from strong $[13,14]$ to absent [15] anisotropies is evidenced, depending on the system. Here we choose $J_{\text {nnn }}=1$, which leads to practically isotropic configurations (see Appendix B).

Our microscopic model with threefold ground-state degeneracy is derived from the $L 1_{2}$ structure. For the composition of $\mathrm{Cu}_{2} \mathrm{NiZn}$ it is known (see Ref. [41] and the references given therein) that its $L 1_{2}$ structure, where $\mathrm{Zn}$ occupies one of the four sublattices on the fcc lattice while $\mathrm{Cu}$ and $\mathrm{Ni}$ jointly occupy the three others, shows a further ordering transition as temperature is decreased, in that the $\mathrm{Ni}$ atoms congregate on one of the three $\mathrm{Cu} / \mathrm{Ni}$ sublattices (Fig. 1). The resulting structure of tetragonal symmetry can equally be seen as a superstructure on $L 1_{0}$ and is thus denoted as "modified" $L 1_{0}$ structure. It has space group $123(P 4 / \mathrm{mmm})$ with $\mathrm{Zn}$ on Wyckoff position (1a), Ni on (1c), and $\mathrm{Cu}$ on (2e). At this transition, the $\mathrm{Zn}$ atoms are unaffected, so it can be expected that within the coarse APD structure of the $L 1_{2}$ superstructure formed at higher temperature, a fine $\mathrm{m}-L 1_{0}$ APD structure will result as temperature is decreased, with threefold degeneracy with respect to a given $L 1_{2}$ domain. In our model we disregard the static $\mathrm{Zn}$ atoms. The situation with respect to the anisotropy of the APD boundary energy is analogous to the
$L 1_{2}$ case, and also here we use $J_{\mathrm{nnn}}=1$ to get approximately isotropic interfacial energies.

\section{B. Abstract models}

For the standard ferromagnetic $q$-state Potts model [42], the Hamiltonian reads

$$
H=-J \sum_{\langle i, j\rangle} \delta_{\sigma_{i}, \sigma_{j}},
$$

where $\delta_{\sigma_{i}, \sigma_{j}}$ denotes the Kronecker delta, and the spin variables take $q$ distinct values. Again, the nearest-neighbor interaction constant $J$ is chosen as the (positive) unit of energy.

\section{Implementation and dynamics}

We implemented the B1 and Potts cases on simple cubic $N \times N \times N$ lattices, while the face-centered cubic lattice on which the $L 1_{2}$ and $\mathrm{m}-L 1_{0}$ superstructures develop is implemented as $N / 2 \times N / 2 \times N / 2$ four-site conventional cubic fcc cells. In all cases we used $N=1024$. The pseudorandom numbers used in the applied algorithms were generated by the permuted congruential generator [43]. Unless stated otherwise, our coarsening domain structures are initialized from a completely random state, apart from fixing exact stoichiometry in the Ising case.

In our microscopic Ising models, the temporal evolution of the configuration is driven by the random movement of a vacancy according to the Metropolis algorithm [44]: at each step, a neighboring site is randomly selected, and the difference in the number of bonds between equal and unequal atoms on nearest and next-nearest neighbors is used as index into a precomputed two-dimensional table of exchange probabilities. Comparing another random number to these Metropolis exchange rates

$$
p(\Delta H)= \begin{cases}1, & \Delta H<0, \\ \exp \left(-\frac{\Delta H}{k_{\mathrm{B}} T}\right), & \Delta H \geqslant 0,\end{cases}
$$

decides whether the exchange is actually performed. For the $L 1_{2}$ and $\mathrm{m}-L 1_{0}$ cases, which are asymmetric with respect to $\mathrm{A}$ and $\mathrm{B}$ atoms, the vacancy interacts with the other atoms as if it was a majority atom. Thus, a proposed exchange with a majority atom will always be performed, which prevents an otherwise conceivable trapping of the vacancy in locally optimal configurations. Note that, apart from being physically more realistic, the good data locality of vacancy dynamics makes it also computationally more efficient as measured in terms of CPU cycles per attempted site occupation exchange due to the cache architecture of modern CPUs, while the socalled Kawasaki dynamics of attempted exchanges of random pairs of neighboring atoms $[45,46]$ is bounded by the latency of accessing unpredictable locations in main memory and thus is significantly slower, in particular for large system sizes.

In the abstract Potts models, site updates happen via the heat bath algorithm [47]: Considering a given site, first a histogram of the spin states of its neighbors is constructed. These frequencies of neighboring spin states can be translated to the canonical probabilities for the state of the considered 
site by table look-up and normalization. Finally, a new state (not necessarily different from the original) is assigned according to these probabilities. In order to avoid introducing dynamical and thus potentially structural anisotropies due to sequential updating of sites, we exploit the bipartite nature of the simple-cubic lattice and conceptually update alternatingly the white and black sites of a corresponding three-dimensional checkerboard pattern, as sites of given color do not directly interact among themselves.

Time is measured in units of Monte Carlo steps (MCS), where one MCS corresponds to as many attempted vacancy moves as there are sites in the system in case of the microscopic models, while in the Potts models every spin is reassigned exactly once per MCS. In the coarsening simulations, we followed the evolution of the configurations over 7500 MCS.

We run our simulations on a workstation with four processor threads. In the Potts cases we exploit parallel execution by dividing the set of white or black sites along one dimension into eight slices and update the even- followed by the odd-numbered slices concurrently. We use FFTW [48] for computing the reciprocal-space scattering functions and realspace correlation functions, and, for being able to handle the data volume, we reduce these three-dimensional quantities to one-dimensional radial averages on the fly during the simulation. For each system we performed from 40 up to 80 of these coarsening simulations. In the quantitative analysis we compute the reported expected values of the derived parameters from the correlation functions and scattering functions averaged over these independent configurations. The estimated errors are derived via the bootstrap method [49], that is, by repeatedly generating synthetic samples of configurations with the same number of elements by sampling with replacement from the simulations, performing the analysis on the corresponding averaged correlation or scattering functions, and computing the standard deviation thereof.

\section{Equilibrium properties}

In order to decide on the temperature for the $L 1_{2}$ and $\mathrm{m}-L 1_{0}$ coarsening simulations, as well as to check our implementations by comparing to the known phase transition temperatures in the Potts cases, we computed the temperaturedependent long-range order parameter $S(T)$. Disregarding APDs for now, we use the definition

$$
S=c_{\alpha}^{\mathrm{A}}-c_{\beta}^{\mathrm{A}}=c_{\beta}^{\mathrm{B}}-c_{\alpha}^{\mathrm{B}},
$$

corresponding to the difference of the occupation of the sublattices. On the other hand, for the $q$-state Potts model we use

$$
S=\frac{q x-1}{q-1},
$$

where $x$ is the proportion of the majority spin state. Obviously, assuming stoichiometric composition in the microscopic case, both expressions vary between 0 (for the totally disordered) and 1 (for the totally ordered case).

To start with the case $q=2$ in the Potts model, slowly increasing the temperature on an initially perfectly ordered system leads to a continuous decrease of the order



FIG. 2. Temperature-dependent order parameters $S\left(k_{\mathrm{B}} T\right)$ of the Potts (a) and microscopic systems (b). Uncertainties are generally much smaller than the symbol sizes.

parameter (Fig. 2), reaching zero at about $k_{\mathrm{B}} T_{\mathrm{c}}=2.255(2)$. This reproduces the well-known behavior of the threedimensional ferromagnetic $q=2$ Potts case, and also our estimate for the transition temperature is quantitatively consistent with the best currently known value $k_{\mathrm{B}} T_{\mathrm{c}}=2.25576163(5)$ [50], obtained by methods that are much more efficient for second-order transitions than our simple local Monte Carlo updates. As mentioned already, by flipping the sign of the interaction and the occupation of the sites of one color in the three-dimensional checkerboard decomposition, the microscopic B1 case becomes thermodynamically equivalent, and indeed the order parameter curves perfectly coincide when scaling the temperature by a factor of 2 due to the respective choice of units, in particular our transition temperature of $k_{\mathrm{B}} T_{\mathrm{c}}=4.510(2)$ is consistent with the Potts value.

Our simulations for the $q=3$ and $q=4$ Potts models show a discontinuous transition at $k_{\mathrm{B}} T_{\mathrm{c}}=1.8164(1)$ and 1.5907(1), respectively. Again, this is qualitatively and quantitatively consistent with the most precise reported transition temperatures of $k_{\mathrm{B}} T_{\mathrm{c}}=1.816315(20)$ and 1.590816(9) [51]. Note that determining the transition temperatures simply by continuously increasing the temperature would lead to inaccurate values due to the necessary nucleation of the disordered phase, thus in these cases we determined the transition temperatures by starting with an inhomogeneous system consisting of an ordered and a disordered part and adjusting the simulation temperature until the two phases are seemingly in equilibrium, indicated by the order parameter showing a purely stochastic evolution with simulation time. The presented curves have been obtained by simulations on heating and on cooling, spliced together at the critical temperature obtained as detailed above.

The order parameter curves for the microscopic $q=3$ and $q=4$ cases display an additional detail: while at a first glance they seem to qualitatively follow the discontinuous 
behavior of the corresponding Potts cases, actually they show an approximately linear decrease over the regions $k_{\mathrm{B}} T \in$ $(1.814,1.830)$ in case of $q=3$ and $k_{\mathrm{B}} T \in(1.668,1.680)$ in the $q=4$ case. This is due to the conservation of particle numbers in the microscopic models: as is familiar from binary alloy phase diagrams, different phases are typically separated by two-phase regions. In general, due to the details of thermodynamics in the two phases at elevated temperatures there is no reason for a congruent transition to happen exactly at stoichiometry. Note that this is different for $q=2$, where the above-mentioned symmetry fixes the congruent transition at stoichiometry. Thus, the linear decrease of the order parameter corresponds to a situation of coexisting ordered and disordered regions in the simulation cells, with weights that are varying with temperature, and is representative for the equilibrium behavior of the systems.

\section{DOMAIN COARSENING}

The detailed arrangement of APD boundaries in an actual experimental sample or in a simulation, being the consequence of microscopic nucleation, growth, and coarsening dynamics, is of course different from realization to realization. However, these specific realizations are governed by statistical properties, which can be meaningfully discussed and compared. The simplest of these properties pertain to the APD pair-correlation function $g(\vec{r})$. Apart from its simplicity of definition, the pair-correlation function's importance stems from the fact that it, being a second-order correlation function, can be experimentally directly accessed via the variation of scattered radiation over reciprocal space. For being able to compare pair-correlation functions for different $q$, here we will use a rescaled version that decays from 1 at small distances to 0 at infinity. Specifically, where $g^{\prime}(\vec{r})$ is the conventional pair-correlation function defined as the probability of two random points separated by $\vec{r}$ to be located in the same domain, our rescaled correlation function is defined as

$$
g(\vec{r})=\left[q g^{\prime}(\vec{r})-1\right] /(q-1) .
$$

We give the actual definitions of $g^{\prime}(\vec{r})$ for the different systems in Appendix A.

While atomically sharp APD boundaries resulting at low temperatures in general do show a pronounced dependence of interfacial energy on crystallographic direction and thus lead to strong facetting of the domains with a preference for low-energy domain boundaries (typically specific low-index planes) [52], at elevated temperatures the local degree of order is depressed at the boundaries, the interfaces become diffuse, and consequently the directional dependence of interfacial energies and the anisotropy of the pair-correlation function diminish. For the sake of simplicity, in the following we will report only radially averaged correlation functions $g(r)$. We give an account for the validity of this approximation in Appendix B.

On the other hand, at temperatures close to the transition point defects appear also inside the APDs, which complicate the assignment of sites and their occupations to domains. Instead of coarsening the scale and computing fractional assignments via local averages along the point of view of phase field modeling, here we directly take site occupations, but

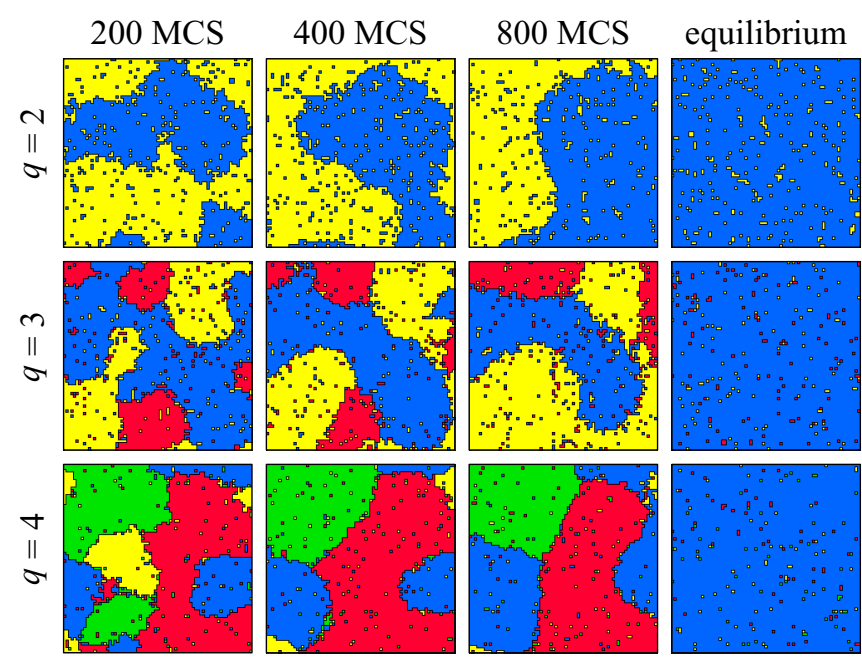

FIG. 3. Coarsening configurations for the three Potts models at various stages, together with final configuration of a single domain with isolated point defects. Shown are sections of 100 sites edge length of two-dimensional slices, while the three-dimensional simulation box is considerably larger.

correct for the effect of long-range order degrees smaller than unity as detailed in Appendix C.

In order to have approximately isotropic APD configurations without introducing too much atomic-scale disorder, we use consistently temperatures of about $75 \%$ of the phase transition temperature $T_{\mathrm{c}}$ for our coarsening simulations. Specifically, we use values for $k_{\mathrm{B}} T$ of $1.7296,1.4$, and 1.2 for the abstract cases of $q=2, q=3$, and $q=4$, and 3.4592, 1.36275 , and 1.26 for the corresponding microscopic cases.

\section{A. Dynamical scaling}

Representative snapshots of the configuration at different times during the coarsening process are depicted in Fig. 3 for the Potts systems with various $q$. Coloring the sites according to their spin state directly shows the domain configuration. The pair-correlation functions $g(r)$ for various coarsening times $t$ are depicted in Fig. 4 (left top), exemplarily for $q=4$. As the domains grow, the correlation length increases, the APD boundary density decreases, and thus $g(r)$ decays slower with distance.

A corresponding behavior is visible in reciprocal space in Fig. 4 (left bottom), where the structure factors $S(k)$ as the pair-correlation functions' Fourier transform display a redistribution of intensity to smaller $k$ indicating longer correlation lengths. A corresponding behavior is observed in all investigated systems.

According to the scaling hypothesis, at late times the correlation functions, and thus also the corresponding structure factors, can be scaled on top of each other. This is also fulfilled in our simulations, as shown exemplarily for the $q=4$ Potts system in Fig. 4 (right column): with a single scaling parameter $L(t)$ both real-space correlation functions and reciprocal-space structure factors can be brought into satisfactory agreement by plotting them as functions 


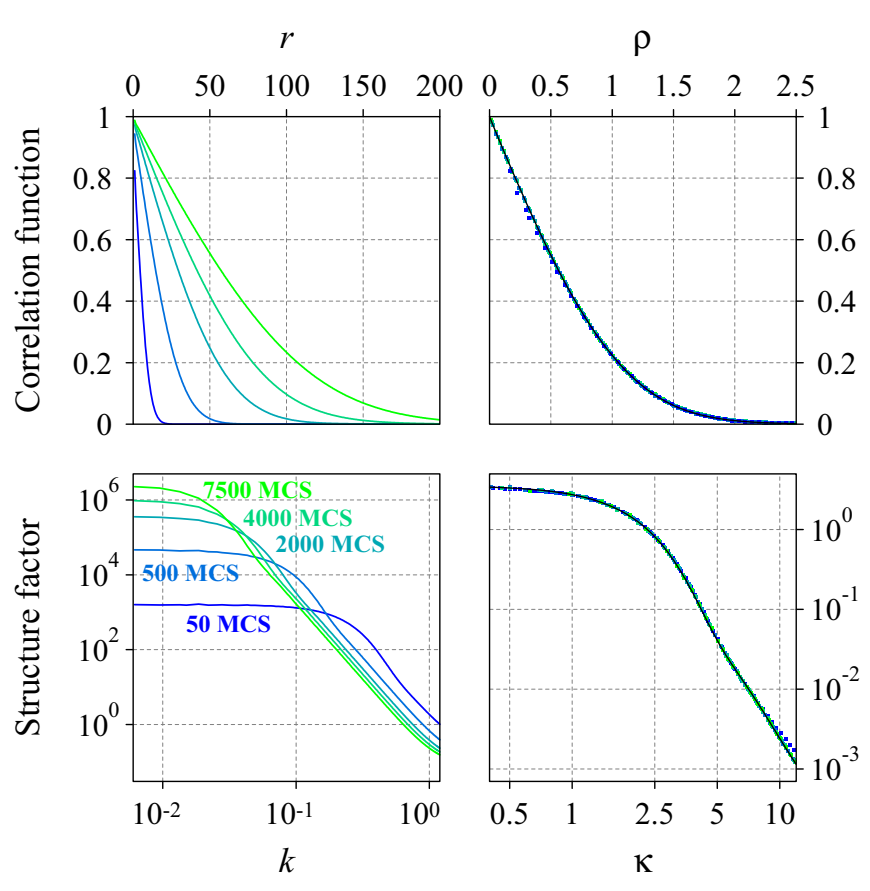

FIG. 4. Simulated radially averaged real-space correlation functions (top left) and reciprocal-space structure factors (bottom left) of $q=4$ Potts model at various coarsening stages. In the right column, the data are replotted on the scaled coordinates together with the scaling functions, demonstrating the universal shape in the scaling regime. Note that only a subset of simulated points is plotted for being able to distinguish the data sets.

of

$$
\begin{gathered}
\rho=r q / L(t)(q-1) \quad \text { and } \\
\kappa=k L(t)(q-1) / q,
\end{gathered}
$$

respectively [the reason for introducing the term $q /(q-1)$ will become clear in Sec. III D]. Only at the earliest times with correspondingly small $L(t)$, effects due to the fundamental discretization of space overlap with the features due to the APD configuration, leading to deviations from scaling at small $\rho$ and large $\kappa$.

\section{B. The scaling function}

\section{Phenomenological ansatz}

To obtain a simple analytical expression for the general form of the scaling function in real as well as in reciprocal space we use an ansatz in terms of exponential decays and Gaussian functions. We have found that a linear combination of an exponential decay and two Gaussian functions for the correlation function adequately reproduces our simulation results:

$$
g(\rho)=b e^{-\rho / b}+\sum_{i=1}^{2} a_{i} e^{-\rho^{2} / 2 \sigma_{i}^{2}} .
$$

The exponential decay at large $\rho$ of this expression conflicts with the expected superexponential decay due to diffusive interaction [17], but we want to note that the asymptotic realspace behavior is practically accessible neither in simulations nor in experiments. In reciprocal space, our expression gives the correct asymptotic behavior both for small and large $\kappa$.

Setting $b=1-a_{1}-a_{2}$ the model fulfills the condition $g(0)=1$ that is necessary for any correlation function. Furthermore, by construction it has a derivative of -1 at $\rho=0$. This latter property allows us to define the spatial scale of a simulated APD configuration via the factor by which its correlation function has to be scaled in order to fit the scaling function via Eq. (7). As we will show in Sec. III D, this implies that the specific interface area of the configuration in the sense of interface area per unit volume is given by $2 / L$. A discussion of how $L$ increases with coarsening time $t$ is given below in Sec. III C.

By the relation for the three-dimensional Fourier transform of radially symmetric functions [53]

$$
[\mathcal{F}(f)](\kappa)=4 \pi \int_{0}^{\infty} d \rho \frac{\sin (\kappa \rho)}{\kappa} f(\rho) \rho,
$$

the expression for the corresponding structure factor is

$$
S(\kappa)=\frac{8 \pi}{\left(\kappa^{2}+1 / b^{2}\right)^{2}}+\sqrt{8 \pi^{3}} \sum_{i=1}^{2} a_{i} \sigma_{i}^{3} \exp \left(-\kappa^{2} \sigma_{i}^{2} / 2\right) .
$$

Furthermore, integrating this three-dimensional radially symmetric function over two perpendicular dimensions gives the expression corresponding to the peak profile in powder diffractometry

$$
S^{\prime}\left(\kappa_{x}\right)=\frac{8 \pi^{2}}{\kappa_{x}^{2}+1 / b^{2}}+\sqrt{32 \pi^{5}} \sum_{i=1}^{2} a_{i} \sigma_{i} \exp \left(-\kappa_{x}^{2} \sigma_{i}^{2} / 2\right),
$$

which is remarkably similar to the phenomenological pseudoVoigt profiles typically used for this purpose.

\section{Fitting the scaling function}

We determine the parameters of Eq. (9) by fitting the scaled simulated real-space correlation functions at the successive coarsening time steps for all six of our considered systems. Specifically, we first restrict the (unscaled) correlation functions to the region $r \geqslant 10$ in units of the simple-cubic lattice constant to exclude short-range order effects. We then minimize the weighted squared deviations between the scaled simulated correlation functions and the model curve, varying concurrently the common model parameters $a_{1}, a_{2}, \sigma_{1}, \sigma_{2}$ as well as the specific scaling parameters $L(t)$. We use weights so that after rescaling a given interval in $\rho$ contributes according to its width in logarithmic units, considering the range $0.1 \leqslant \rho \leqslant 5$, analogously for the coarsening time in the range $25 \leqslant t \leqslant 7500$ MCS. In our view, this is the most efficient way to use the available information in the simulations, as essentially new configurations are attained after the scale parameter $L$ has increased by a given factor, which under a power-law behavior of $L(t)$ corresponds to a given logarithmic increment in coarsening time $t$.

\section{Results and theoretical expressions}

The resulting real-space and reciprocal-space scaling functions for the $q=4$ Potts case are given in the right column of Fig. 4 along with the scaled simulation results. Obviously the 
TABLE I. Parameters of the scaling function given in Eq. (9) and the corresponding full width at half maximum $B_{\mathrm{FWHM}}$ and integral breadth $B_{\mathrm{I}}$ of the powder-diffraction intensity given in Eq. (12).

\begin{tabular}{|c|c|c|c|c|c|c|c|}
\hline \multirow[t]{2}{*}{$q=2$} & Potts & $0.0557(106)$ & $0.6296(242)$ & $0.2841(47)$ & $0.6280(27)$ & $4.058(16)$ & $5.113(20)$ \\
\hline & B1 & $0.0428(60)$ & $0.5805(139)$ & $0.2905(71)$ & $0.6240(34)$ & $4.064(17)$ & $5.137(17)$ \\
\hline \multirow{2}{*}{$q=3$} & Potts & $0.0419(67)$ & $0.5506(172)$ & $0.2930(59)$ & $0.6609(31)$ & $3.852(25)$ & $4.928(24)$ \\
\hline & $\mathrm{m}-L 1_{0}$ & $0.0609(62)$ & $0.5634(161)$ & $0.3170(57)$ & $0.6499(65)$ & $3.952(44)$ & $5.033(45)$ \\
\hline$q=4$ & Potts & $0.0450(37)$ & $0.5253(90)$ & $0.3126(30)$ & $0.6860(21)$ & $3.726(17)$ & $4.804(17)$ \\
\hline
\end{tabular}

agreement is very good, also for the reciprocal-space structure factor, even though only the real-space correlation functions are used for fitting.

The parameters of the scaling functions for all systems are listed in Table I, where for convenience we also give the full widths at half maximum $B_{\text {FWHM }}$ and the integral breadth $B_{\mathrm{I}}$ of the corresponding powder diffractometry peak profiles.

A comparison of the scaling functions for the various systems is given in the left column of Fig. 5. Even though the systems qualitatively differ on the microscopic scale, the simulated correlation functions and structure factors look sur-
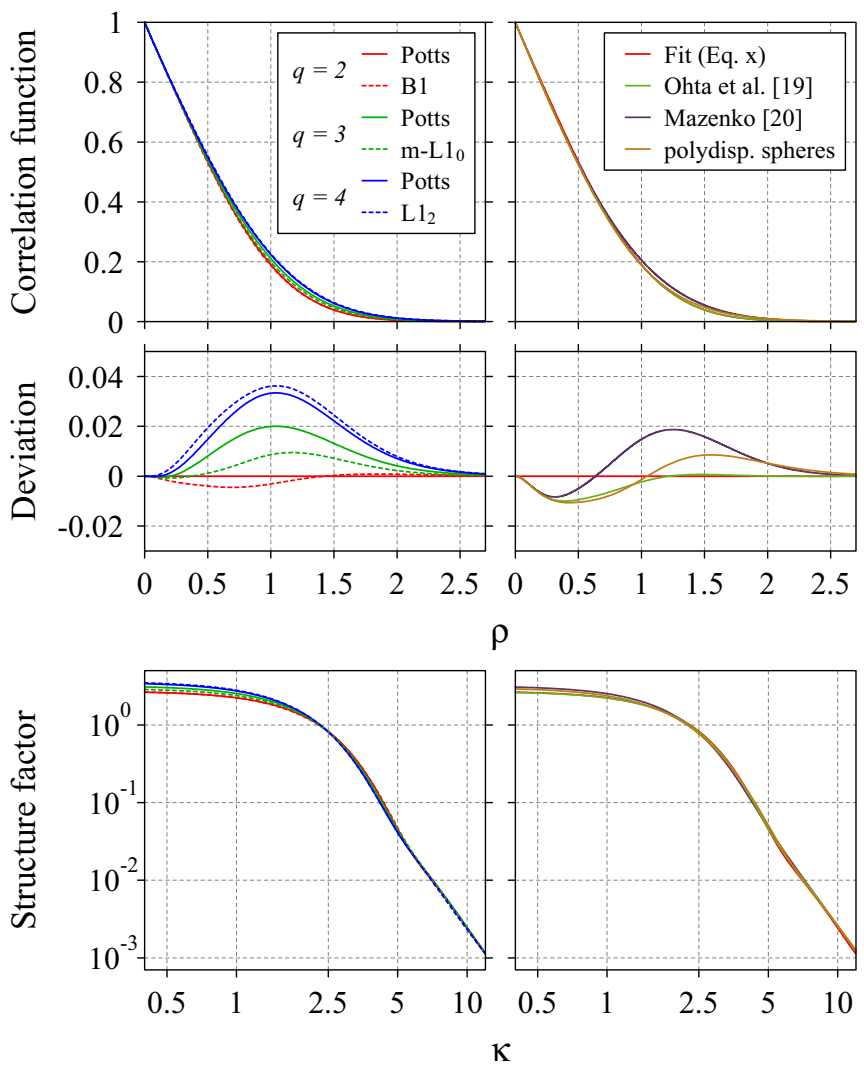

FIG. 5. Scaling functions for the real-space correlation functions (top) and reciprocal-space structure factors (bottom), comparing our phenomenological functions according to Eqs. (9) and (11) for the Potts and microscopic systems for various $q$ (left column) and various theoretical predictions for $q=2$ to our corresponding Potts functions. To highlight the differences between the systems and theories, the middle row shows the deviations with respect to our $q=2$ Potts correlation function. prisingly similar. Computing the deviations of the correlation functions shows that, as expected, the main differences are between systems of different $q$, while Potts and microscopic systems for the same $q$ typically show much less deviations. An exception is the case $q=3$, where the $\mathrm{m}-L 1_{0}$ correlation function is about halfway between the $q=3$ Potts and the $q=$ 2 cases. In particular, for a given initial slope of the correlation function, the correlations at intermediate distances increase with increasing $q$. We think that this is due to the APD boundary edges present for $q \geqslant 3$ and corners for $q \geqslant 4$ : if we construct a $q$-state APD configuration by first dividing space into a large number of randomly arranged compact regions, and then randomly assign a state from 1 to $q$ to each region, the correlation function will be independent of $q$. However, if we then allow this configuration to coarsen, for $q=2$ it will quickly evolve smooth boundaries, thus decreasing the interfacial area and leading to increased correlations at small $\rho$, while for larger $q$ edges and corners will evolve, giving a comparatively smaller increase at small $\rho$.

To decide on the significance of these findings, we turn to a statistical evaluation: in Table II we give the $L^{2}$ distances between the different fitted phenomenological correlation functions together with their estimated statistical error as obtained by bootstrap sampling (thus, the first row quantifies the deviations as plotted in Fig. 5). There we see that the deviations between systems of different $q$ are indeed in most cases highly significant different from zero. On the other hand, the deviations between Potts and microscopic systems for the same $q$ are also typically a factor of 2 larger than the corresponding expected errors, where the above-noted $q=3$ case is no exception, but only shows a larger estimated error. Thus, our simulations are still consistent with the hypothesis of universal scaling functions for given $q$, also taking into account systematic effects due to different weights of possible early-time deviations from scaling, but do show significant deviations for different $q$.

Analytic predictions for the form of the scaling function in the $q=2$ case were given by Ohta and co-workers, the OJK model [24], as well as by Mazenko [25]. The right column of Fig. 5 compares these functions with the simulated $q=2$ Potts case. The visual agreement is quite good, but given the fact that the variation with $q$ is small according to the simulations, the OJK model performs significantly better than the Mazenko model, where the latter even seems to be more representative for the $q=3$ case than for $q=2$. To test also an expression modeling a very different situation, we turn to spatially uncorrelated polydisperse spheres: a log-normal distribution of sphere radii with parameters that fulfill the 
TABLE II. The $L^{2}$ distance between the real-space correlation functions of the different systems, along with estimated statistical errors.

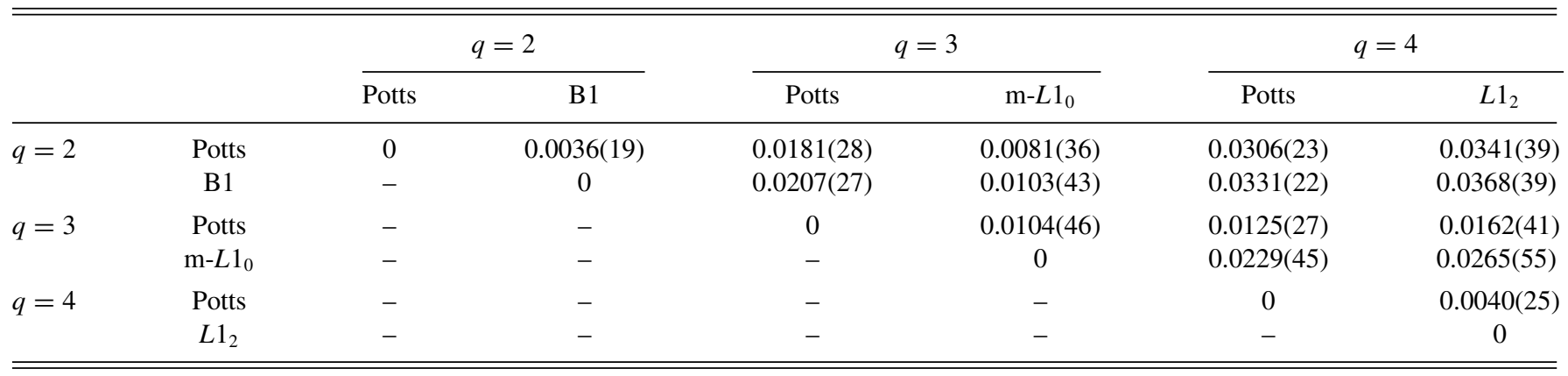

condition $\mu=-5 / 2 \sigma^{2}+\log (3 / 4)$ gives the correct specific interface area. Choosing $\sigma=0.3$ as a single free parameter leads to the correlation function and structure factor displayed in Fig. 5-obviously it is about as successful as the dedicated models in describing the simulations. A systematic effect common to all theoretical expressions is an underestimation of the simulated correlation function at small distances $\rho$, as can be seen in the deviation plot.

\section{Kinetics}

The evolution of the APD scale during coarsening for the various systems is given in Fig. 6. The classical prediction for the increase of the spatial scale in the simplest case of $O(1)$ symmetry would be $L(t) \propto t^{1 / 2}$ [16], and the same should hold for Potts cases [54]. Note that the absolute zero of time has to be treated as a free parameter here, as it is not guaranteed that the length scale with which the simulation enters the scaling regime is consistent with the time when it does so according to the scaling relation. Thus, we have shifted the data in time, typically by positive values of a few MCS, to achieve a small- $t$ behavior as systematic as possible.

In our simulations the Potts systems seem to follow the expected $t^{1 / 2}$ behavior quite well, but a closer look reveals

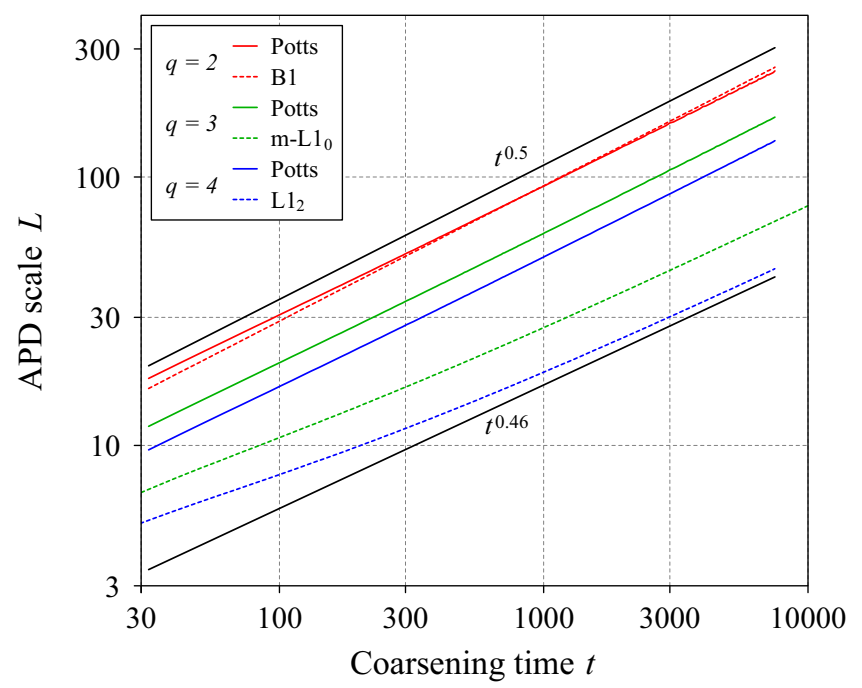

FIG. 6. Growth of the characteristic length scales $L$ with time for the various systems, together with lines indicating the behaviours $L(t) \propto t^{0.5}$ and $L(t) \propto t^{0.46}$. that the best fit is achieved by slightly reduced exponents of about 0.48 . Such a behavior has already been reported $[31,55]$ and suggested to be due to either a pinning of defects on the lattice or the asymptotic regime having not yet been reached [31]. As our values pertain to much larger lattices and longer simulations, we feel save to rule out the latter option.

The microscopic systems show a different behavior. On the one hand, the B1 system exhibits a growth exponent of about 0.505 , slightly but significantly larger than $1 / 2$. We conjecture that this is due to an energetical attraction of the vacancy to the APD boundaries, where it is more effective in driving coarsening [56]: as the specific interface area decreases with simulation time, the constant vacancy content of the simulation box would lead to an increasing vacancy concentration in the boundaries and thus faster coarsening $[57,58]$.

On the other hand, the $\mathrm{m}-L 1_{0}$ and $L 1_{2}$ systems show striking deviations from a power-law behavior. This can be due either to a transition between regimes or to logarithmic corrections to power-law scaling - the instantaneous growth exponent seems to increase monotonously, reaching about 0.46 towards the final stages of the simulation. In these cases, we suspect the effect of conservation laws to be responsible: while in all microscopic models the particle numbers are conserved, the symmetry of the B1 case rules out any systematic deviation from 1:1 stoichiometry at the APD boundaries. In contrast, for $\mathrm{m}-L 1_{0}$ and $L 1_{2}$ there is no argument against segregation of one or the other component towards the APD boundaries. Thus, the coarsening of APD configurations will entail some long-range diffusion of atoms.

The Potts cases differ only in $q$, thus their kinetic behavior can be directly compared. We observe that it takes longer to reach a given APD scale $L$ with increasing $q$. The dominant effect for this behavior is easily understandable: in the above-mentioned construction of a model APD configuration by assigning random states to compact regions, the probability for neighboring regions to belong to the same domain is larger for smaller $q$, thus giving a larger APD scale. Also the microscopic cases suggest a qualitatively corresponding behavior, but we have to note here that due to the microscopic differences of the lattices, the definitions of the domain scales cannot be compared, thus the observed behavior should be regarded as fortuitious.

\section{Measures of domain size}

By construction, our scaling function for the real-space correlation function $g(\rho)$ decreases with a derivative of -1 
at small distances. In the following we will derive to which measure of real-space APD scale the corresponding scaling parameter $L$ corresponds.

Consider two points A and B randomly located in threedimensional space and separated by a distance $r$, and assume a specific interfacial area of $\sigma$ (that is the average area of interface per unit volume). If now all these interfaces were oriented perfectly perpendicular to the vector connecting A to $\mathrm{B}$, then the probability of this vector to pass through one interface would be equal to $\sigma r$ in the limit of small $r$. For arbitrary orientations of the interfaces this has to be multiplied by a factor $1 / 2$, which is the mean absolute value of a randomly oriented three-dimensional unit vector's projection along a given direction. Thus, in this limit the probability for $\mathrm{A}$ and $\mathrm{B}$ to be in the same domain, which is nothing else than the (unscaled) correlation function $g^{\prime}(r)$, is given by

$$
g^{\prime}(r) \approx 1-\sigma r / 2
$$

As a consequence, with Eqs. (6) and (7) the scaling parameter $L$ that leads to $g(\rho)$ having a derivative of -1 at $\rho=0$ fulfills

$$
L=2 / \sigma \text {. }
$$

A main point to note is that our measure for the APD scale $L$ is exactly equivalent to the linear intercept method [10] that is typically used for quantitative evaluations of APD sizes in microscopy investigations (e.g., Refs. [7-9]), where the number of intersections along a path in the image plane is counted and related to the path length-assuming isotropic APD configurations, any two points along the path can take the role of A and B in the previous paragraph. Indeed, in our opinion it would be more appropriate to consistently use "specific interface area" or "interfacial density" for this quantity rather than "average APD size," as it is often sloppily called (note that, for not too large $q$, covering the cases of practical interest, the APD configuration is percolating in three dimensions, thus a domain's average size in some strict sense of the word, be it the volume or linear extent, is not defined).

The most basic approach to quantitatively analyze diffraction data with respect to the size of the coherently diffracting domains is afforded by the Scherrer equation [11], which relates the width of the diffraction peaks $\Delta k$ in units of the wave-vector transfer to the scale of real-space features $\bar{D}$,

$$
\bar{D}=\frac{2 \pi K}{\Delta k},
$$

neglecting in this simplest form any contributions due to instrumental resolution or strain to the broadening. $K$ is a phenomenological constant of order unity that depends on the real-space model of the system and thus the corresponding definition of $\bar{D}$, and on how the peak broadening $\Delta k$ is measured. Specifically, assuming monodisperse spherical domains of volume $\bar{D}^{3}$ in real space, which is the most basic choice for the case of powders or polycrystals, and determining $\Delta k$ as the FWHM of the peak implies $K_{\mathrm{FWHM}} \approx$ 0.8920 [59], while using the integral breadth leads to $K_{\mathrm{I}}=$ $\sqrt[3]{32 \pi / 81}=1.0747$ [60]. In default of a microscopic model, often also for studies of APD coarsening the real-space scales have been reported by employing Eq. (15) with $K$ set to unity (e.g., Ref. [61]). As we have shown that the scaling functions are universal for given $q$, the values we give for the FWHM and integral breadth of the peak shape in Table I now allow us to relate scales determined by microscopy and diffraction quite easily, obviating the need for a detailed phenomenological modeling in terms of distribution functions [62,63].

To be specific, defining $\bar{D}=L=2 / \sigma$ in Eq. (15) leads to Scherrer constants $K_{\mathrm{FWHM}}=1.2916$ and $K_{\mathrm{I}}=1.6275$ for the values corresponding to the Potts $q=2$ scaling function. With increasing $q$, the dominant effect determining the behavior of $K$ is the factor $2(q-1) / q$ relating the derivative in $g(r)$ to $\sigma$, while the shapes of the scaling functions are of only secondary importance, leading to $K_{\mathrm{FWHM}}=0.7908$ and $K_{\mathrm{I}}=1.0195$ for the $q=4$ Potts case. Interestingly, the ratio of the scale defined by $L$ to the cubic root of spherical domains is $\sqrt[3]{16 / 9 \pi} \approx 0.8271$, assuming volume-filling spheres. Thus, taking into account the specific definitions for the spatial scale $\bar{D}$ the above-mentioned Scherrer constants of order unity indeed will give an acceptable approximation to the $q=\infty$ case, corresponding to the polycrystalline case, while for the low $q$ with APD configurations they would lead to a drastic overestimation of the specific interface area.

\section{SUMMARY}

We have studied the coarsening of domain configurations in statistical models with discrete symmetry breaking and nonconserved order parameter by large-scale Monte Carlo simulations, considering not only the well-studied $O(1)$ Ising case, but also symmetry breakings with $q=3$ and 4 groundstate degeneracy. Apart from the abstract Potts (Ising) models, we considered also corresponding realistic models of atomic ordering in compounds. The primary results of our simulations are the radially varying real-space correlation functions and reciprocal-space structure factors.

After initial periods of a few tens of Monte Carlo steps, we observe perfect scaling of the correlation functions and structure factors over more than two orders of magnitude in time, or one order of magnitude in spatial scale. As expected, in general we found an increase of the spatial scale with the square root of time, while the subtleties of the atomistic models lead to deviations that are clearly resolvable thanks to the good statistics of our simulations.

We propose a simple ansatz for the real-space and reciprocal-space scaling functions, and we give the parameters that result in a very good fit of to the simulation results. While the differences between the models are remarkably small, the good statistics of our simulations allow us to conclude that the scaling functions vary significantly with $q$, but not among the abstract and atomistic models for the same $q$. Interestingly, the deviations the $q=2$ simulations and different theoretical predictions are on the same order of magnitude as those between simulations for different $q$.

Finally, we demonstrated the equivalence of our definition of the scale parameter with the standard linear intercept method for obtaining domain sizes in microscopy, being essentially the inverse of the specific interface area. Thus, our results establish a quantitative link between domain sizes measured via the peak broadening in diffraction and those measured in microscopy without any adjustable parameters. 


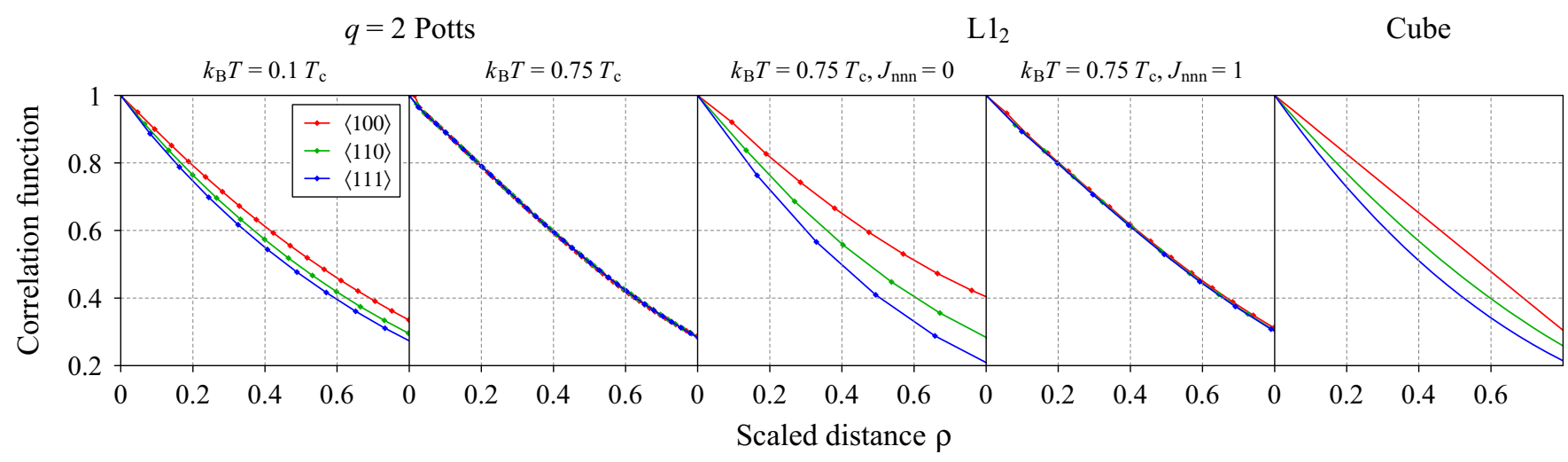

FIG. 7. Direction-dependent pair-correlation functions: $q=2$ Potts system at $k_{\mathrm{B}} T=0.1 T_{\mathrm{c}}$ and $0.75 T_{\mathrm{c}}, L 1_{2}$ system with $J_{\mathrm{nnn}}=0$ and $J_{\mathrm{nnn}}=1$, all after $1500 \mathrm{MCS}$ apart from $L 1_{2}$ system with $J_{\mathrm{nnn}}=0$, which due to its slow coarsening was simulated for $30000 \mathrm{MCS}$, compared with the pair-correlation function of a cube.

\section{ACKNOWLEDGMENT}

Financial support by the Deutsche Forschungsgemeinschaft (Project No. 107745057, TRR 80) is gratefully acknowledged.

\section{APPENDIX A: DEFINITIONS OF THE PAIR-CORRELATION FUNCTION}

The conventional APD pair-correlation function $g^{\prime}(r)$ is defined as the probability for two random points separated by a distance $r$ to be in the same domain. In the abstract Potts models, the state of a given spin directly corresponds to the domain the corresponding site belongs to. However, for the microscopic systems, the situation is not so obvious. We use the following definitions: For computing $g^{\prime}(r)$ for the $\mathrm{m}-L 1_{0}$ and $L 1_{2}$ cases, we consider each of the three or four, respectively, simple-cubic sublattices separately, compute the pair-correlation function of the minority atoms, and sum the contributions. Ideally, exactly one of the three or four sites per unit cell is occupied by a minority atom, and a given unit cell belongs to domain $i$ if it is its site $i$ that is occupied by a minority atom. Under point-defect disorder, in our definition a given unit cell can belong to zero, one, or more domains. As a consequence of treating the simple-cubic sublattices independently, the smallest accessible distance corresponds to the cubic lattice constant. On the other hand, in the B1 case we conceptually cover the system by a threedimensional checkerboard pattern, exchange the spins on the, say, white sites by their opposite, and compute the conventional pair-correlation function. This approach allows to probe also nearest-neighbor distances.

\section{APPENDIX B: ISOTROPY OF APD STRUCTURES}

In order to test for anisotropy in the statistical properties of the APD configurations, we performed dedicated coarsening simulations on smaller systems and evaluated directionally dependent correlation functions in $\langle 100\rangle,\langle 110\rangle$, and $\langle 111\rangle$ directions as reported in Fig. 7. As expected, at the low simulation temperature of $0.1 T_{\mathrm{c}}$ strong directional differences on the order of $10 \%$ result for the exemplarily considered $q=2$ Potts case, while the more diffuse interfaces at the simula- tion temperature of $0.75 T_{\mathrm{c}}$, which we use in the remainder of this work, lead to differences that are visually vanishing. As a second example we considered the microscopic $L 1_{2}$ case. For exclusively nearest-neighbor interactions when setting $J_{\mathrm{nnn}}=0$, even at $0.75 T_{\mathrm{c}}$ (with $T_{\mathrm{c}}$ for the corresponding model taken from Ref. [39]) strong anisotropies are visible, but again our choice of second-nearest-neighbor interactions $J_{\mathrm{nnn}}=-1$ leads to isotropic features in a very good approximation, which is our justification for assuming isotropic APD configurations in the main part of this work.

In both anisotropic cases considered here, the initial decay of the correlation function is steepest along the $\langle 111\rangle$ directions, followed by $\langle 110\rangle$. This is consistent with a preference for $\{100\}$-oriented APD boundaries, which can be explained on the one hand by the $\{100\}$ planes being the close-packed planes in the simple cubic lattice that hosts the Potts systems, while, as discussed in Sec. II A, in the $L 1_{2}$ case with $J_{\mathrm{nnn}}=0$ for two given APDs there is a specific plane of $\{100\}$ type that can host a locally stoichiometric interface without an associated cost in energy. Indeed, these cases are already about as anisotropic as possible under cubic symmetry: assuming as an extreme model an APD configuration where three-dimensional space is tesselated by a lattice of cubes and the volume enclosed by a given cube is assigned to a random antiphase domain, the resulting correlation function can be calculated analytically as the autocorrelation function of a single cube-shaped domain

$$
g(\rho)= \begin{cases}\max (0,1-\rho) & \text { along }\langle 100\rangle, \\ \max (0,1-\rho / \sqrt{2})^{2} & \text { along }\langle 110\rangle, \\ \max (0,1-\rho / \sqrt{3})^{3} & \text { along }\langle 111\rangle\end{cases}
$$

As can be seen in Fig. 7 , in particular the case of an $L 1_{2}$ system with exclusively nearest-neighbor interactions gives anisotropies as pronounced as this most extreme model.

\section{APPENDIX C: CORRECTING FOR DISORDER}

In the case of a perfectly ordered system the correlation function is unity. Due to our using a comparatively high ordering temperature, which is necessary for diffuse APD boundaries and thus approximately isotropic configurations, we have significant concentrations of point defects also away 


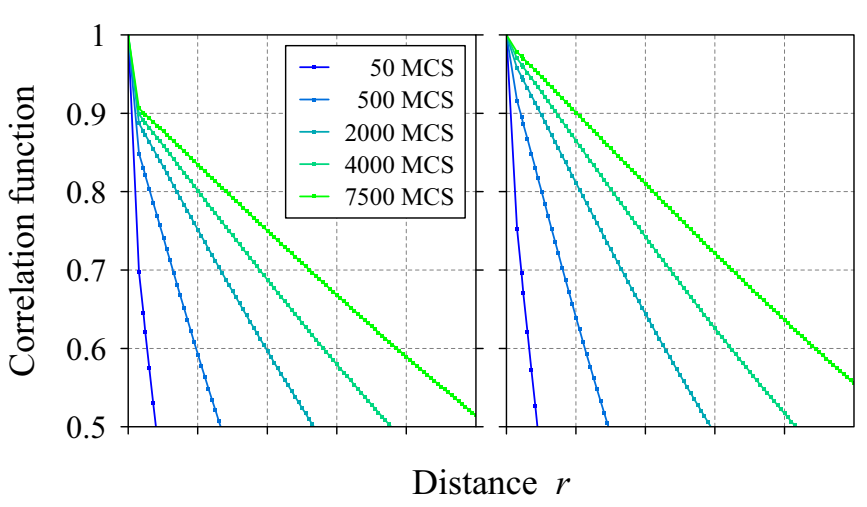

FIG. 8. APD correlation functions of the $q=4$ Potts model in the fully uncorrected version (left), and corrected for disorder according to Eq. (C3) (right).

from the APD boundaries. Here we specify how we derive the correlation function of the idealized APD configuration corresponding to a given configuration of the spins subject to thermal disorder.

We start with the effect due to a finite long-range order parameter, giving point defects away from APD boundaries. We distinguish between the underlying APD correlation function $g^{\prime}(r)$ unaffected by thermal disorder and the apparent APD correlation function $f^{\prime}(r)$ determined as detailed in Appendix A, which is reduced compared to $g^{\prime}(r)$ due to thermal disorder. Let $p(r)$ be the probability that two points separated by a distance $r$ are assigned to the same domain in equilibrium, that is, when the whole system in principle belongs to one domain, but where point defects lead to corresponding defects in the domain assignment. We can then write the uncorrected APD correlation function in a coarsening simulation as

$$
f^{\prime}(r)=g^{\prime}(r) p(r)+\left[1-g^{\prime}(r)\right][1-p(r)] /(q-1) .
$$

Here we made the approximation that the correlations of point defects as quantified by $p(r)$ behave across APD boundaries as they do within a domain, which will be exactly fulfilled in the limit of vanishing point defect correlations, that is, at large distances. Solving for $g^{\prime}(r)$ gives

$$
g^{\prime}(r)=\frac{f^{\prime}(r)(q-1)-1+p(r)}{q p(r)-1} .
$$

Rescaling according to Eq. (6) gives the particularly simple expression

$$
g(r)=\frac{q f^{\prime}(r)-1}{q p(r)-1} .
$$

For simplicity, and justified by the fact that in fitting we anyway consider the correlation functions only for $r \geqslant 10$ in order to avoid effects due to short-range order of defects, we replace $p(r)$ by its long-distance limit, given by the concentration of point defects in equilibrium as determined in dedicated simulations.

The effect of the correction for thermal point defects is demonstrated in Fig. 8. While the improvement is obvious, a close look shows that the expected behavior of an extrapolation to exactly 1 as $r \rightarrow 0$ is still not fulfilled. This is due to the depression of order close to the APD boundaries, where the introduction of a point defect is obviously less costly than in the interior of the domains. In the simplest approach we can model the APD boundaries as having a finite width $d$, within which the order is completely lost. As the specific interface area is $2 / L$, the extrapolation of the correlation function to $r=0$ will correspondingly be reduced from 1 to $1-2 d / L$. Due to the correlation function's derivative of $-2 / L$ at 0 , we correct for this effect by shifting the distance scale $r$ by $d$. The fitted values for $d$ with increasing $q$ are $0.75,0.62$, and 0.51 for the Potts cases, and $0.76,0.71$, and 0.57 for the microscopic systems.
[1] N. S. Stoloff, Int. Metals Rev. 29, 123 (1984).

[2] T. M. Pollock and S. Tin, J. Propul. Power 22, 361 (2006).

[3] P. Neibecker, M. E. Gruner, X. Xu, R. Kainuma, W. Petry, R. Pentcheva, and M. Leitner, Phys. Rev. B 96, 165131 (2017).

[4] A. E. Vidoz, D. P. Lazarević, and R. W. Cahn, Acta Metall. 11, 17 (1963).

[5] R. C. Reed, The Superalloys: Fundamentals and Applications (Cambridge University Press, Cambridge, UK, 2006).

[6] R. G. Davies and N. S. Stoloff, Acta Metall. 11, 1347 (1963).

[7] J. A. Rogers, H. M. Flower, and R. D. Rawlings, Metal Sci. 9 , 32 (1975).

[8] A. Suzuki, M. Takeyama, and T. Matsuo, Intermetallics 10, 915 (2002).

[9] F. E. Brenker, W. F. Müller, and G. P. Brey, Am. Mineral. 88, 1300 (2003).

[10] C. S. Smith and L. Guttman, J. Metals 5, 81 (1953).

[11] P. Scherrer, Nachr. Ges. Wiss. Göttingen, Math.-Phys. K1. 2, 98 (1918).

[12] P. Scardi and M. Leoni, Acta Crystallogr. 58, 190 (2002).

[13] M. Sakai and D. E. Mikkola, Metall. Trans. 2, 1635 (1971).
[14] D. G. Morris, F. M. C. Besag, and R. E. Smallman, Philos. Mag. A 29, 43 (1974).

[15] D. G. Morris, G. T. Brown, R. C. Piller, and R. E. Smallman, Acta Metall. 24, 21 (1976).

[16] S. M. Allen and J. W. Cahn, Acta Metall. 27, 1085 (1979).

[17] A. J. Bray, Adv. Phys. 51, 481 (2002).

[18] S. Katano and M. Iizumi, Phys. Rev. Lett. 52, 835 (1984).

[19] H. Toyoki, Phys. Rev. B 45, 1965 (1992).

[20] A. Guinier and G. Fournet, Small-Angle Scattering of X-Rays (Wiley \& Sons, New York, 1955), pp. 12-16.

[21] G. Porod, Kolloid-Z. 124, 83 (1951).

[22] I. Grillo, Colloids Surf. A 225, 153 (2003).

[23] K. Kawasaki, M. C. Yalabik, and J. D. Gunton, Phys. Rev. A 17, 455 (1978).

[24] T. Ohta, D. Jasnow, and K. Kawasaki, Phys. Rev. Lett. 49, 1223 (1982).

[25] G. F. Mazenko, Phys. Rev. B 42, 4487 (1990).

[26] F. Liu and G. F. Mazenko, Phys. Rev. B 44, 9185 (1991).

[27] F. Rojas and A. D. Rutenberg, Phys. Rev. E 60, 212 (1999). 
[28] V. L. Berezinskii, Zh. Eksp. Teor. Fiz. 59, 907 (1970); Sov. Phys. JETP 32, 493 (1971).

[29] J. M. Kosterlitz and D. J. Thouless, J. Phys. C 6, 1181 (1973).

[30] M. K. Phani, J. L. Lebowitz, M. H. Kalos, and O. Penrose, Phys. Rev. Lett. 45, 366 (1980).

[31] R. E. Blundell and A. J. Bray, Phys. Rev. E 49, 4925 (1994).

[32] F. Y. Wu, Rev. Mod. Phys. 54, 235 (1982).

[33] R. Vetter and C. M. van Baal, Philos. Mag. B 61, 189 (1990).

[34] A. J. Bradley, A. H. Jay, and W. L. Bragg, Proc. R. Soc. A 136, 210 (1932).

[35] S. M. Allen and J. W. Cahn, Acta Metall. 23, 1017 (1975).

[36] P. Pochet, E. Tominez, L. Chaffron, and G. Martin, Phys. Rev. B 52, 4006 (1995).

[37] C. Frontera, E. Vives, T. Castán, and A. Planes, Phys. Rev. B 55, 212 (1997).

[38] S. E. Nagler, R. F. Shannon, C. R. Harkless, M. A. Singh, and R. M. Nicklow, Phys. Rev. Lett. 61, 718 (1988).

[39] M. Kessler, W. Dieterich, and A. Majhofer, Phys. Rev. B 67, 134201 (2003).

[40] M. Kessler, W. Dieterich, and A. Majhofer, Physica A 330, 25 (2003).

[41] S. I. Simak, A. V. Ruban, I. A. Abrikosov, H. L. Skriver, and B. Johansson, Phys. Rev. Lett. 81, 188 (1998).

[42] R. B. Potts, Proc. Cambridge Philos. Soc. 48, 106 (1952).

[43] M. E. O'Neill, PCG: A Family of Simple Fast Space-Efficient Statistically Good Algorithms for Random Number Generation, Tech. Rep. HMC-CS-2014-0905 ( Harvey Mudd College, Claremont, CA, 2014).

[44] N. Metropolis, A. W. Rosenbluth, M. N. Rosenbluth, A. H. Teller, and E. Teller, J. Chem. Phys. 21, 1087 (1953).

[45] K. Kawasaki, Phys. Rev. 145, 224 (1966).
[46] A. B. Bortz, M. H. Kalos, J. L. Lebowitz, and M. A. Zendejas, Phys. Rev. B 10, 535 (1974).

[47] M. Creutz, Phys. Rev. D 21, 2308 (1980).

[48] M. Frigo and S. G. Johnson, Proc. IEEE 93, 216 (2005), special issue on "Program Generation, Optimization, and Platform Adaptation".

[49] B. Efron and R. J. Tibshirani, An Introduction to the Bootstrap, Chapman \& Hall/CRC Monographs on Statistics \& Applied Probability (CRC, Boca Raton, FL, 1994).

[50] A. M. Ferrenberg, J. Xu, and D. P. Landau, Phys. Rev. E 97, 043301 (2018).

[51] A. Bazavov, B. A. Berg, and S. Dubey, Nucl. Phys. B 802, 421 (2008).

[52] D. Le Floc'h, P. Bellon, and M. Athènes, Phys. Rev. B 62, 3142 (2000).

[53] A. Iosevich and E. Liflyand, The Fourier Transform of a Radial Function, in Decay of the Fourier Transform (Springer, Basel, 2014), pp. 93-126.

[54] A. D. Rutenberg and A. J. Bray, Phys. Rev. E 51, 5499 (1995).

[55] G. S. Grest, M. P. Anderson, and D. J. Srolovitz, Phys. Rev. B 38, 4752 (1988).

[56] P. Fratzl and O. Penrose, Phys. Rev. B 50, 3477 (1994).

[57] E. Vives and A. Planes, Phys. Rev. Lett. 68, 812 (1992).

[58] C. Frontera, E. Vives, and A. Planes, Z. Phys. B 96, 79 (1994).

[59] A. L. Patterson, Phys. Rev. 56, 978 (1939).

[60] J. I. Langford and A. J. C. Wilson, J. Appl. Crystallogr. 11, 102 (1978).

[61] R. Gilles, M. Hofmann, Y. Gao, F. Johnson, L. Iorio, M. Larsen, F. Liang, M. Hölzel, and B. Barbier, Met. Mater. Trans. A 41, 1144 (2010).

[62] G. Sauthoff, Acta Metall. 21, 273 (1973).

[63] M. Leoni and P. Scardi, J. Appl. Crystallogr. 37, 629 (2004). 